\title{
High antibiotic prescription rates in hospitalized children with human metapneumovirus infection in comparison to RSV infection emphasize the value of point-of-care diagnostics
}

\author{
D. Schreiner ${ }^{1}$ (D) B. Groendahl ${ }^{1} \cdot$ W. Puppe ${ }^{2} \cdot$ H. Naraghi Taghi Off ${ }^{3} \cdot$ K. Poplawska $^{1} \cdot$ M. Knuf $^{1,4} \cdot$ C. U. Meyer ${ }^{1}$. \\ A. T. Reischl ${ }^{1} \cdot$ Stephan Gehring $^{1}$
}

Received: 12 June 2018 / Accepted: 13 August 2018 / Published online: 21 August 2018

(c) Springer-Verlag GmbH Germany, part of Springer Nature 2018

\begin{abstract}
Background Respiratory infections are the main causes for hospitalization in children and a common reason for the initiation of antibiotic treatment. Rapid antigen detection tests and point-of-care mPCR-based assays provide a fast detection of viral pathogens. Nonetheless, the prescription rate of antibiotics for respiratory infections is exceedingly high. In particular, human metapneumovirus (hMPV) infections frequently cause antibiotic treatment.

Methods Children hospitalized in our clinic with an acute respiratory infection between January 2008 and January 2013 were included in the present study. Data of 3799 children were analyzed retrospectively for clinical symptoms, laboratory findings, and antibiotic and inhalation treatment. We performed an in-house m-RT-PCR-ELISA method for pathogen detection. Results Pathogen detection was possible in 2464 patients. In 6.3\%, hMPV and, in 24.0\%, RSV were detected. Patients positively tested for hMPV received inhalation therapy in $62.9 \%$; patients positive for RSV in $73.8 \%$. Patients positive for hMPV were treated with antibiotics in $62.3 \%$. Patients with RSV infection received antibiotic treatment in $44.4 \%$; all others in $43.5 \%$. Notably, a positive result in RSV-RADT was associated with reduced number of antibiotic treatment.

Conclusion hMPV infections inherit a two times higher probability of antibiotic treatment. There was no significant difference in laboratory findings or body temperature between hMPV infection and infections caused by other pathogens. Clinical symptoms seem not to differ from those in RSV illness. Nonetheless, RSV infections triggered significantly lower antibiotic prescription rates. A considerate application of a POC-mPCR for patients with RSV-like symptoms and age of 1 year and older with a negative RSV-RADT might lead to higher detection rates of hMPV and a reduction in prescription of antibiotics.
\end{abstract}

Keywords Human metapneumovirus · hMPV $\cdot$ RSV $\cdot$ Antibiotic treatment · Point-of-care diagnostic

\section{Introduction}

Respiratory infections are main causes for hospitalization in children and a common reason for antibiotic treatment $[1,2]$. Rapid antigen detection tests (RADT) and point-ofcare (POC) multiplex polymerase chain reaction (mPCR)based assays provide a fast, and in case of the latter, reliable detection of viral pathogens. POC diagnostic tools have been reported to reduce the prescription of antibiotic drugs [3, 4]. Nonetheless, the prescription rate of antibiotics for respiratory infections in Germany is still higher as recommended by the European Surveillance of Antimicrobial Consumption project (ESAC) [5].

Human metapneumovirus was first described in 2001 [4]. It belongs to the family of Paramyxoviridae, the subfamily of Pneumovirinae, and the genus Metapneumovirus [6]. It

Children's Hospital, Helios Dr. Horst Schmidt Clinic Wiesbaden, Wiesbaden, Germany 
is closely related to the Respiratory Syncytial Virus (RSV), which is member of the same subfamily but of the different genus Pneumovirus [6]. Patients, who suffer from hMPV infection for the first time, showed symptoms resembling RSV illness. At the age of 5 years, nearly all tested children showed a positive serology for hMPV; however, reinfection has been reported [7].

Besides a close phylogenetic relationship of hMPV and RSV [7, 8], clinical symptoms are very similar and rated as indistinguishable [6,9]. Just like RSV, hMPV can cause uncomplicated infections of the upper airway up to severe bronchiolitis and community-acquired viral pneumonia [10-14].

According to preexisting studies, hMPV can be detected as pathogen in 5-14\% of pediatric patients with symptoms of an acute respiratory infection $[11,12,14]$.

In our single-center study, we analyzed data of 3799 children and found hMPV to trigger most frequently antibiotic treatment compared to all other viral pathogens. The aim of this study was to identify characteristic features of hMPV infections providing a rationale to reduce the prescription of antibiotic drugs.

\section{Methods}

Children admitted to the Center for Pediatric and Adolescent Medicine at the Mainz University Medical Center with an acute respiratory infection between January 2008 and January 2013 were included in the present study. Data of 3799 children were retrospectively analyzed for clinical findings, laboratory parameters, antibiotic therapy, and inhalation treatment. By admittance to inpatient treatment, consent was given to anonymized scientific analysis of the data acquired during diagnostics and treatment.

Material for diagnostics was obtained from every patient in the emergency department during admission to inpatient treatment. It was collected by nasopharyngeal swap or commercially provided suctioning systems. As recently described, an in-house developed multiplex reverse transcriptase polymerase chain reaction combined with a microwell hybridization assay (m-RT-PCR-ELISA) was performed for pathogen detection [15]. The following 13 viruses and 5 bacterial pathogens could be detected from a single sample: enterovirus, influenza virus type A and type $B$, respiratory syncytial virus (RSV), parainfluenza virus type 1 , type 2 , type 3 , and type 4 , adenovirus, rhinovirus, human metapneumovirus (hMPV), coronavirus, reovirus, Mycoplasma pneumoniae, Chlamydophila pneumoniae, Bordetella pertussis, Bordetella parapertussis, and Legionella pneumophila. M-RT-PCR-ELISA was performed weekdays, when a certain number of samples had accumulated and could be processed in one run. From sample collection to the final result of the m-RT-PCR-ELISA, it took in average 3 working days. RSV and influenza RADT (Certest Biotec S.L.) were performed as POC diagnostic for every patient during admission. The sensitivity for RSV is $95 \%$ and the specificity $>99 \%$. These data are provided by the manufacturer and reflect the results of a comparison of Certest RADT to another RADT. An evaluation by PCR was not provided. The reference RADT has a pooled sensitivity of $81 \%$ and a pooled specificity of $97 \%$ evaluated by PCR and culture [16].

All other data were obtained from our clinical information system.

The data were analyzed with GraphPad Prism 7, Version 7.03 (GraphPad Software Inc.). Statistical significance was evaluated with Fisher's exact test with a confidence interval of 95\%, respectively, the Holm-Sidak method with alpha $=0.05$ for multiple $t$ tests.

\section{Results}

During the period from January 2008 to January 2013, a total of 3799 samples were analyzed with m-RT-PCRELISA. Nasal secretions derived from 2464 children with signs of lower respiratory tract (LRT) infections had a positive m-RT-PCR-ELISA result. In the subsequent analysis, we present the results of all patients with at least one identified pathogen $(n=2464)$.

The median age was 18 months (IQR 35 months). A total of $1429(58.0 \%)$ were male.

Rhinovirus was detected most frequently with a total of 1267 patients (51.4\%). In 155 patients (6.3\%), hMPV and, in 592 (24.0\%), RSV were detected. Both, hMPV and RSV displayed a very similar and distinct seasonal pattern with an increased incidence from November until April. hMPV showed a higher incidence in 1 year followed by lower incidence the next year (Fig. 1). This is in accordance to the published data [17]. While other authors found the highest incidence between February and March [11], we found its peaking in parallel to RSV season in 2009 and 2010. In 2008 (not completely shown) and 2012, hMPV occurred before RSV season, and in 2011, most hMPV infections were detected between March and May.

RSV-infected children were significantly younger than children with hMPV infection. For RSV, the median age was 9 vs. 21 months in hMPV infection (Fig. 2; Table 1). There was no difference in the length of hospital stay (7 days in RSV vs. 7.1 days in hMPV).

As mentioned before, the symptoms of hMPV infection cannot easily be discriminated from RSV infection. They have even been described as undistinguishable $[6,9]$. To validate clinical symptoms, we compared the frequency of inhalation therapy and the application of 


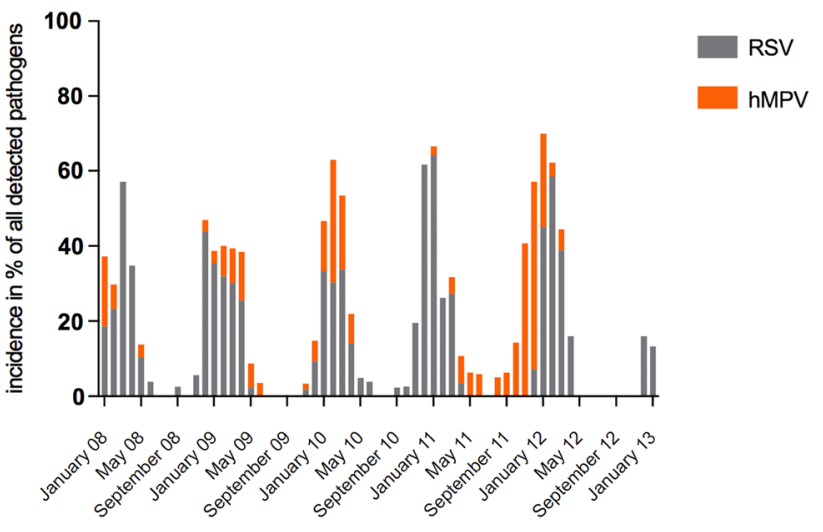

Fig. 1 Seasonal distribution of RSV and hMPV. The figure shows the detection rates for RSV and hMPV in percent of all the detected pathogens for each month from January 2008 to January 2013

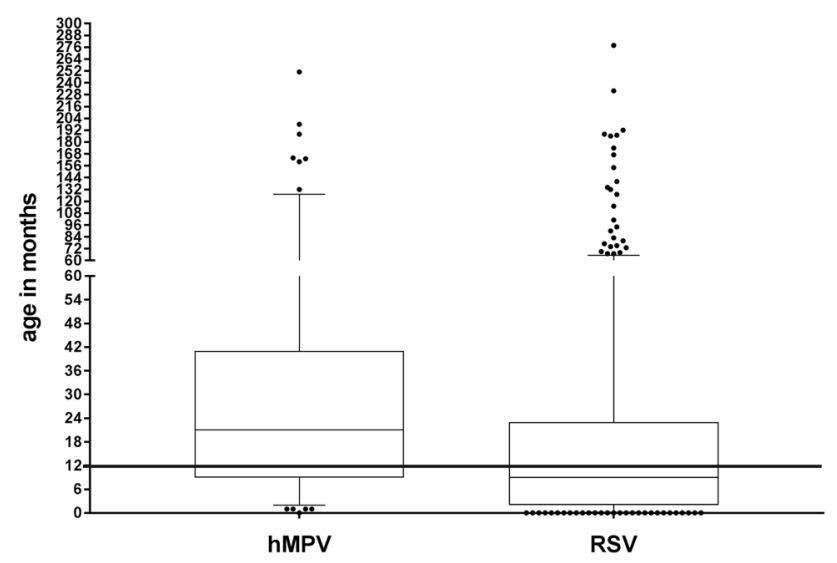

Fig. 2 Age of the patients with hMPV and RSV infection. The figure shows the median and the interquartile range of the patients' age suffering from hMPV or RSV infection. The whiskers show the 95\% CI. The black line is our proposed cutoff at 12 months for POC-mPCR

systemic steroids as a surrogate for pulmonary obstruction (Fig. 3; Table 2). The prescription of inhaled salbutamol, a combination of salbutamol and ipratropium bromide, or epinephrine inhalations is summarized in the following sections as "inhalation therapy". Patients positively tested for $\mathrm{hMPV}$ received inhalation therapy in $62.9 \%$, in contrast to $73.8 \%$ of patients positive for RSV. Compared to patients with respiratory infections other than hMPV or RSV, who received inhalation therapy in $42.6 \%$, the odds ratio (OR) for receiving this treatment was significantly higher with 2.3 (1.6-3.2) for hMPV and 3.8 (3.1-4.7) for RSV infection, respectively. When comparing hMPV and RSV directly, there was a slightly higher chance of being treated with inhalation therapy if a patient was positively tested for RSV in m-RT-PCR-ELISA with an OR of 1.7 (0.6-2.4).

The laboratory parameters including white blood cell counts (WBC) and the C-reactive protein (CRP) did not differ between hMPV, RSV, and the median of the other respiratory viruses tested (Table 1).

All patients included in this study received RADT for RSV. $66 \%$ of the RSV-positive children in m-RT-PCRELISA had a positive RSV-RADT. Patients with hMPV in m-RT-PCR-ELISA showed a positive RSV-RADT only in 11 out of 155 (7\%). Not taking the m-RT-PCR-ELISA testing into account, $41.7 \%$ of all patients with a positive RSV-RADT received antibiotics, in contrast to $49.6 \%$ with antibiotic treatment and negative RSV-RADT result. With an OR of 0.73 (0.59-0.89), this is a significant reduction in antibiotic therapy associated with the positive RSV-RADT result (see Table 3).

As outlined above, results of the m-RT-PCR-ELISA were available in average 3 days after the nasal specimens were obtained. Thus, m-RT-PCR-ELISA results did not influence the initial treatment regimen of these patients.

Notably, antibiotic treatment differed significantly between the groups of evaluated pathogens. Patients positive for hMPV in m-RT-PCR-ELISA were treated with antibiotics in $62.3 \%$. Patients with RSV infection detected by $\mathrm{m}$-RT-PCR-ELISA received antibiotic treatment in $44.4 \%$; all others in $43.5 \%$. Compared to the latter, a patient with a positive hMPV result was more than two times more likely to receive antibiotic therapy, with an OR of 2.14 (1.52-3.00). There was no significant difference between RSV and the remaining pathogens (Table 2; Fig. 3).
Table 1 CRP, WBC, body temperature, and age in patients with hMPV, RSV, or another respiratory infection

\begin{tabular}{|c|c|c|c|c|c|c|}
\hline & \multicolumn{2}{|l|}{ hMPV } & \multicolumn{2}{|l|}{ RSV } & \multicolumn{2}{|c|}{ All other pathogens } \\
\hline & Median & IQR & Median & IQR & Median & IQR \\
\hline CRP (mg/l) & 9.4 & $0-29$ & 7.9 & $0-23$ & 9.4 & $0-28$ \\
\hline WBC (/nl) & 10.1 & $7.42-14.4$ & 9.67 & $7.5-12.6$ & 11.8 & $8.48-16.1$ \\
\hline Temperature $\left({ }^{\circ} \mathrm{C}\right)$ & 38.5 & $37.4-39.03$ & 38 & $37.3-39$ & 38 & $37.2-39$ \\
\hline Age (months) & 21 & $9-41$ & 9 & $2-23$ & 22 & $9-51$ \\
\hline
\end{tabular}

The table shows the median and interquartile range (IQR) for C-reactive protein (CRP), white blood cell counts (WBC), body temperature, and age at admission to inpatient treatment in children with hMPV or RSV infection or another of the detectable pathogens 


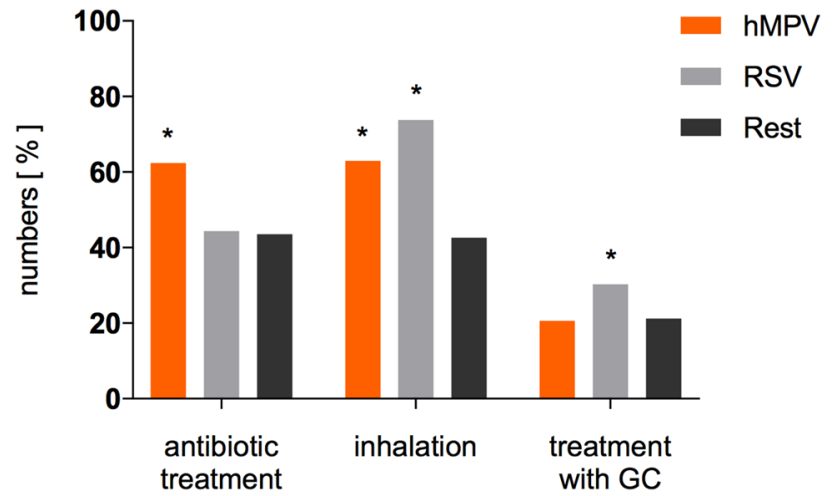

Fig. 3 Frequency of antibiotic treatment, inhalation, and GC treatment. The figure shows the percentage of patients treated with antibiotics, inhalation, or glucocorticoids (GC) depending on the detected pathogen. The asterices indicate the level of significance $\left({ }^{*} p<0.0001\right)$ when comparing hMPV or RSV to the remaining detected pathogens

\section{Discussion}

Between January 2008 and January 2013, 3799 nasal secretions from hospitalized children with acute LRT infection were investigated by m-RT-PCR-ELISA and RADT. Clinical and laboratory data of 2464 patients with a positive m-RT-PCR-ELISA result were collected additionally. To our knowledge, this is one of the largest evaluated cohorts of hospitalized children with acute respiratory tract infection [18-22].

The material collection was done per nasal swab or nasopharyngeal aspirate (NPA). NPA has the highest yield for the detection of respiratory viruses, even higher than in bronchoalveolar lavage [23]. Nasal swab has a moderate decreased sensitivity in virus detection than NPA. In general, the detection rates differ for each respiratory virus but are especially high in RSV-infected patients [24]. The detection rates for common (Streptococcus pneumoniae and Haemophilus influenzae) and atypical bacterial pathogens (M. pneumoniae and C. pneumoniae) are comparable between NPA and nasal swab [25, 26].

Overall, $44.8 \%$ of those children were treated with antibiotics. 1075 of 2400 children (44.8\%) with viral infections received antibiotic treatment. In only 64 out of $2464(0.03 \%)$, bacterial pathogens could be detected by m-RT-PCR-ELISA. However, the typical bacterial pathogens for LRT infections, S. pneumoniae, H. influenzae, and Moraxella catarrhalis, cannot be detected by the applied PCR panel and the result of our m-RT-PCR-ELISA was not available when the decision for or against antibiotic therapy was made.

In accordance to recent reports, $6.3 \%$ of the study population was found positive for hMPV [12, 14]. hMPV infections hold a two times higher probability of antibiotic treatment compared to other viral infections. RSV infections were significantly less frequently treated with antibiotics in our cohort.

hMPV is often compared to RSV, due to its close phylogenetic relation and the similarity of symptoms as airway obstruction and bronchiolitis. Hospitalized RSV-infected children tended to be younger than those with hMPV infection, which is a commonly observed age pattern [9]. We saw a nearly parallel occurrence concerning the seasonal distribution as described before [13]. More precisely, hMPV occurred shortly before, parallel or shortly after RSV season between November and April. Other studies observed that hMPV occurs mainly after the RSV infection season $[9,27]$.

In the present study, no differences could be observed in inflammation parameters, e.g. CRP, WBC, or body temperature, between hMPV, RSV, and the median of other pathogens. Airway obstruction caused by RSV and hMPV was more pronounced compared to the other investigated pathogens. Interestingly, RSV was more frequently treated with inhalation and systemic steroids then hMPV. This might be confounded by our in-house standard protocol requiring inhalation with epinephrine and hypertonic saline in children with a positive RSV-RADT and may not reflect the clinical appearance of the patients. Epinehphrine inhalations were shown to reduce symptoms of bronchiolitis, especially in

Table 3 Antibiotic therapy depending on RSV rapid test

\begin{tabular}{lll}
\hline & \multicolumn{2}{l}{ Antibiotic therapy } \\
\cline { 2 - 3 } & Yes (\%) & No (\%) \\
\hline Positive RSV rapid test & 41.74 & 58.26 \\
Negative RSV rapid test & 49.60 & 50.40 \\
\hline
\end{tabular}

The calculated OR is 0.73 with a $95 \%$ CI of $0.59-0.892$ with a $p$ value of $p=0.002$
Table 2 OR and CI calculated by comparing hMPV, RSV, and all other pathogens

\begin{tabular}{lllllr}
\hline & $\begin{array}{l}\text { All other pathogens } \\
\text { OR }\end{array}$ & $\begin{array}{l}\text { hMPV } \\
\text { OR }\end{array}$ & 95\% CI & $\begin{array}{l}\text { RSV } \\
\text { OR }\end{array}$ & \multicolumn{1}{l}{$95 \%$ CI } \\
\hline Inhalation & 1.00 (Ref) & $2.29 *$ & $1.62-3.21$ & $3.80 *$ & $3.09-4.68$ \\
Glucocorticoid treatment & 1.00 (Ref) & 0.96 & $0.63-1.43$ & $1.61 *$ & $1.303-1.99$ \\
Antibiotic treatment & 1.00 (Ref) & $2.14 *$ & $1.52-3.00$ & 1.04 & $0.86-1.25$ \\
\hline
\end{tabular}

The shown asterisk indicates the level of significance: $* p<0.0001$ 
the first $24 \mathrm{~h}$, but had no influence on the length of hospital stay $[28,29]$. In contrast, there was no recommendation concerning the antibiotic therapy in RSV infection in our in-house protocol.

As mentioned above, the symptoms of hMPV and RSV infection are described as undistinguishable [6,9], whereas other studies see more bronchiolitis in children with RSV infection and more pneumonia in hMPV-positive children $[30,31]$. Moe et al. adjusted clinical, laboratory, and radiographic findings to the age of the patients which led to the disappearance of all detected differences [31]. Since children with RSV infections are significantly younger than those with hMPV infection, the diagnosis of bronchiolitis seems more common, whereas elevated CRP values, wheezing, and the radiologic signs of pneumonia are more frequent in patients with hMPV infection. Regarding all discussed studies, there was no difference in the severity of disease between hMPV and RSV determined by oxygen therapy, length of hospital stay, and admission to intensive care unit $[9,30,31]$.

Taking all this into account, it is highly interesting why children presenting themselves with comparable symptoms are treated differently. Keeping in mind that a positive RSV-RADT led to the increased prescription of inhalation therapy, we assume that it might have also been related to a reduced rate of antibiotic treatment. A positive result of an RSV-RADT was associated significantly with reduced prescription of antibiotics with an OR of $0.73(0.59-0.89)$. This is a clear reduction of the antibiotic prescription rate by a positive RSV-RADT. Practitioners seem to be more inclined to start a symptomatic therapy with inhalations and to refrain from antibiotic treatment, if clinical presentation of the patient and RADT both indicates RSV infection. Antibiotic therapy in RADT negative patients might be triggered by uncertainty of the etiology of the infection and not only by the severity of symptoms or laboratory findings.

Of course, antibiotic therapy might also be triggered by assumed bacterial coinfection, which is well described for RSV infection [32,33]. For hMPV, only one study indicates that superinfection with $S$. pneumoniae can occur, since a pneumococcal vaccine reduced hospitalization of children with hMPV-associated pneumonia [34]. Bacterial coinfection, even in children presenting themselves with wheezing, has been estimated to range from 18 up to $55 \%$ [35-37]. However, it is not possible to draw a clear distinction between bacterial infection and colonization. Neither detection of bacterial pathogens and clinical and radiological signs of pneumonia, nor the decision for antibiotic treatment and positive bacterial cultures could be correlated positively to in other studies [36]. High serum levels of CRP (exceeding 40-60 mg/L) might indicate a bacterial infection [38]. In RSV infection, also lower concentrations of CRP can hint at bacterial coinfections, since RSV is known to suppress the production of proinflammatory proteins [36, 39]. A high viral load of RSV was associated with decreased CRP serum concentration [36]. hMPV lack these genes coding for proteins, which allow RSV to reduce the hosts immune response $[8,40]$. In contrast, lower concentrations of interleukins were found in children infected with hMPV than in children suffering from RSV infection [41]. This underlines the challenges in decision-making concerning antibiotic treatment in children with respiratory tract infections. In our study, antibiotic treatment was likely triggered by elevated laboratory values indicating inflammation (e.g., CRP — which was particularly pronounced in children with adenovirus infections), and/or by clinical symptoms often associated with bacterial infections, like crackles on auscultation. Crackles, regarded as hallmark of pneumonia, are found in hMPV and RSV infections in 35-38\% [30].

All results mentioned above might be confounded by asymptomatic carriers of viral pathogens or children with persistent detectable nucleic acids after a viral infection weeks or even months ago. This is well described for adenoviruses, rhinoviruses, and coronaviruses [42, 43], whereas, for hMPV, for RSV and parainfluenza viruses, a positive result in PCR-diagnostic strongly indicates that the detected pathogen is causative for the acute infection [42].

A certain limitation of our study is the fact that a bacterial superinfection could not be completely ruled out. A further limitation includes the lack of details about preexisting illnesses, radiographic findings, or need for oxygen.

The particular value of the present study is the high number of included patients, m-RT-PCR-ELISA for pathogen detection in every of those patients, and the long observation period over 5 years.

In our clinic, the application of another multiplex PCRbased point-of-care testing, which detects viral and bacterial pathogens within $1 \mathrm{~h}$, has been increasingly used during inpatient treatment. Preliminary data in a small number of patients with hMPV infection support our hypothesis that a reduction in antibiotic prescription might be achieved through an implementation of POC-mPCR (data not published).

In addition, mPCR grants new knowledge about putative common viral infections by providing epidemiological data and the possibility to correlate the patient's clinical presentation to the detected pathogens.

As a consequence of these findings, we do not recommend to perform POC-mPCR in every patient with an LRT infection, since an unselective testing is associated with significant expenses without necessarily influencing clinical decision-making $[44,45]$. In contrast, we suggest the implementation of algorithms helping to increase pretest probability and to identify children in which a positive mPCR result prevents antibiotic therapy. According to our data, children presenting themselves during winter season 
with RSV-like symptoms like cough, airway obstruction, and crackles on auscultation, age of 1 year and older, and a negative RSV-RADT qualify for a PCR-based point-of-care testing. Regarding Fig. 2 a cutoff at 12 months should allow to detect as many children as possible suffering from hMPV infection and those children false negative in RSV-RADT. This might lead to a reduction in antibiotic therapy. Possibly, a shorten length of antibiotic treatment and an improvement in rates of cross-infections during inpatient treatment can be achieved, as well.

\section{Compliance with ethical standards}

Conflict of interest On behalf of all authors, the corresponding author states that there is no conflict of interest.

\section{References}

1. van Horne B, Netherton E, Helton J, Fu M, Greeley C. The scope and trends of pediatric hospitalizations in Texas, 2004-2010. Hosp Pediatr. 2015;5:390-8.

2. Luca M de, Dona D, Montagnani C, et al. Antibiotic prescriptions and prophylaxis in Italian Children. Is it time to change? Data from the ARPEC project. PLoS One. 2016;11:e0154662.

3. Byington CL, Castillo H, Gerber K, et al. The effect of rapid respiratory viral diagnostic testing on antibiotic use in a children's hospital. Arch Pediatr Adolesc Med. 2002;156:1230-4.

4. Subramony A, Zachariah P, Krones A, Whittier S, Saiman L. Impact of multiplex polymerase chain reaction testing for respiratory pathogens on healthcare resource utilization for pediatric inpatients. J Pediatr. 2016;173:196-201.e2.

5. Schulz M, Kern WV, Hering R, Schulz M, Bätzing-Feigenbaum $\mathrm{J}$. Antibiotikaverordnungen in der ambulanten Versorgung in Deutschland bei bestimmten Infektionserkrankungen in 2009Teil 1 und 2. Berlin: Zentralinstitut für kassenärztliche Versorgung in der Bundesrepublik Deutschland; 2014.

6. Panda S, Mohakud NK, Pena L, Kumar S. Human metapneumovirus: review of an important respiratory pathogen. Int J Infect Dis. 2014;25:45-52.

7. van den Hoogen BG, Jong JC de, Groen J, et al. A newly discovered human pneumovirus isolated from young children with respiratory tract disease. Nat Med. 2001;7:719-24.

8. van den Hoogen BG, Bestebroer TM, Osterhaus ADME, Fouchier RAM. Analysis of the genomic sequence of a human metapneumovirus. Virology. 2002;295:119-32.

9. Wang Y, Ji W, Hao C, Yan YD, Shao X, Xu J. Comparison of bronchiolitis of human metapneumovirus and human respiratory syncytial virus. Acta Virol. 2015;59:98-100.

10. Calvo C, Pozo F, Garcia-Garcia ML, et al. Detection of new respiratory viruses in hospitalized infants with bronchiolitis: a threeyear prospective study. Acta Paediatr. 2010;99:883-7.

11. Garcia-Garcia ML, Calvo C, Martin F, Perez-Brena P, Acosta B, Casas I. Human metapneumovirus infections in hospitalised infants in Spain. Arch Dis Child. 2006;91:290-5.

12. García-García ML, Calvo C, Pozo F, Villadangos PA, PérezBreña P, Casas I. Spectrum of respiratory viruses in children with community-acquired pneumonia. Pediatr Infect Dis J. 2012;31:808-13.

13. Maggi F, Pifferi M, Vatteroni M, et al. Human metapneumovirus associated with respiratory tract infections in a 3 -year study of nasal swabs from infants in Italy. J Clin Microbiol. 2003;41:2987-91.

14. Lu G, Li J, Xie Z, et al. Human metapneumovirus associated with community-acquired pneumonia in children in Beijing, China. J Med Virol. 2013;85:138-43.

15. Puppe W, Weigl J, Grondahl B, et al. Validation of a multiplex reverse transcriptase PCR ELISA for the detection of 19 respiratory tract pathogens. Infection. 2013;41:77-91.

16. Chartrand C, Tremblay N, Renaud C, Papenburg J. Diagnostic accuracy of rapid antigen detection tests for respiratory syncytial virus infection: systematic review and meta-analysis. J Clin Microbiol. 2015;53:3738-49.

17. Rafiefard F, Yun Z, Orvell C. Epidemiologic characteristics and seasonal distribution of human metapneumovirus infections in five epidemic seasons in Stockholm, Sweden, 2002-2006. J Med Virol. 2008;80:1631-8.

18. Goriacko P, Saiman L, Zachariah P. Antibiotic use in hospitalized children with respiratory viruses detected by multiplex polymerase chain reaction. Pediatr Infect Dis J. 2017;34:443.

19. Spaeder MC, Custer JW, Bembea MM, Aganga DO, Song X, Scafidi S. A multicenter outcomes analysis of children with severe viral respiratory infection due to human metapneumovirus. Pediatr Crit Care Med. 2013;14:268-72.

20. Kuchar E, Miskiewicz K, Szenborn L, Kurpas D. Respiratory tract infections in children in primary healthcare in Poland. Adv Exp Med Biol. 2015;835:53-9.

21. Bonfim CM, Nogueira ML, Simas PVM, et al. Frequent respiratory pathogens of respiratory tract infections in children attending daycare centers. J Pediatr (Rio J). 2011;87:439-44.

22. Zhang $X$, Zhang J, Cao A. Epidemiological investigation on respiratory diseases in 1300 children, in Jinan, Shandong. Zhonghua Liu Xing Bing Xue Za Zhi. 2014;35:1275-7.

23. Wurzel DF, Marchant JM, Clark JE, et al. Respiratory virus detection in nasopharyngeal aspirate versus bronchoalveolar lavage is dependent on virus type in children with chronic respiratory symptoms. J Clin Virol. 2013;58:683-8.

24. Blaschke AJ, Allison MA, Meyers L, et al. Non-invasive sample collection for respiratory virus testing by multiplex PCR. J Clin Virol. 2011;52:210-4.

25. Rapola S, Salo E, Kiiski P, Leinonen M, Takala AK. Comparison of four different sampling methods for detecting pharyngeal carriage of Streptococcus pneumoniae and Haemophilus influenzae in children. J Clin Microbiol. 1997;35:1077-9.

26. Meerhoff TJ, Houben ML, Coenjaerts FEJ, et al. Detection of multiple respiratory pathogens during primary respiratory infection: nasal swab versus nasopharyngeal aspirate using real-time polymerase chain reaction. Eur J Clin Microbiol Infect Dis. 2010;29:365-71.

27. Mizuta K, Abiko C, Aoki Y, et al. Seasonal patterns of respiratory syncytial virus, influenza A virus, human metapneumovirus, and parainfluenza virus type 3 infections on the basis of virus isolation data between 2004 and 2011 in Yamagata, Japan. Jpn J Infect Dis. 2013;66:140-5

28. Hartling L, Bialy LM, Vandermeer B, et al. Epinephrine for bronchiolitis. Cochrane Database Syst Rev 2011:CD003123.

29. Black CP. Systematic review of the biology and medical management of respiratory syncytial virus infection. Respir Care 2003;48:209-31 (discussion 231-233).

30. Wolf DG, Greenberg D, Kalkstein D, et al. Comparison of human metapneumovirus, respiratory syncytial virus and influenza $\mathrm{A}$ virus lower respiratory tract infections in hospitalized young children. Pediatr Infect Dis J. 2006;25:320-4.

31. Moe N, Krokstad S, Stenseng IH, et al. Comparing human metapneumovirus and respiratory syncytial virus: viral co-detections, genotypes and risk factors for severe disease. PLoS One. 2017; 12:e0170200. 
32. Stockman LJ, Reed C, Kallen AJ, Finelli L, Anderson LJ. Respiratory syncytial virus and Staphylococcus aureus coinfection in children hospitalized with pneumonia. Pediatr Infect Dis J. 2010;29:1048-50.

33. Thorburn K, Harigopal S, Reddy V, Taylor N, van Saene HKF. High incidence of pulmonary bacterial co-infection in children with severe respiratory syncytial virus (RSV) bronchiolitis. Thorax. 2006;61:611-5.

34. Madhi SA, Ludewick H, Kuwanda L, et al. Pneumococcal coinfection with human metapneumovirus. J Infect Dis. 2006;193:1236-43.

35. Carlsson CJ, Vissing NH, Sevelsted A, Johnston SL, Bønnelykke $\mathrm{K}$, Bisgaard H. Duration of wheezy episodes in early childhood is independent of the microbial trigger. J Allergy Clin Immunol. 2015;136:1208-14.e1-5.

36. Franz A, Adams O, Willems R, et al. Correlation of viral load of respiratory pathogens and co-infections with disease severity in children hospitalized for lower respiratory tract infection. J Clin Virol. 2010;48:239-45.

37. Lehtinen P, Jartti T, Virkki R, et al. Bacterial coinfections in children with viral wheezing. Eur J Clin Microbiol Infect Dis. 2006;25:463-9.

38. Flood RG, Badik J, Aronoff SC. The utility of serum C-reactive protein in differentiating bacterial from nonbacterial pneumonia in children: a meta-analysis of 1230 children. Pediatr Infect Dis J. 2008;27:95-9.
39. Spann KM, Tran K-C, Chi B, Rabin RL, Collins PL. Suppression of the induction of alpha, beta, and lambda interferons by the NS1 and NS2 proteins of human respiratory syncytial virus in human epithelial cells and macrophages corrected. J Virol. 2004;78:4363-9.

40. Schildgen V, van den Hoogen B, Fouchier R, et al. Human metapneumovirus: lessons learned over the first decade. Clin Microbiol Rev. 2011;24:734-54.

41. Laham FR, Israele V, Casellas JM, et al. Differential production of inflammatory cytokines in primary infection with human metapneumovirus and with other common respiratory viruses of infancy. J Infect Dis. 2004;189:2047-56.

42. Rhedin S, Lindstrand A, Rotzen-Ostlund M, et al. Clinical utility of PCR for common viruses in acute respiratory illness. Pediatrics. 2014;133:e538-45.

43. van der Zalm MM, Wilbrink B, van Ewijk BE, Overduin P, Wolfs TFW, van der Ent CK. Highly frequent infections with human rhinovirus in healthy young children: a longitudinal cohort study. J Clin Virol. 2011;52:317-20.

44. Krause JC, Panning M, Hengel H, Henneke P. The role of multiplex PCR in respiratory tract infections in children. Dtsch Arztebl Int. 2014;111:639-45.

45. Gill PJ, Richardson SE, Ostrow O, Friedman JN. Testing for respiratory viruses in children: to swab or not to swab. JAMA Pediatr. 2017;171:798-804. 